\title{
La influencia de la posesión del balón en el rendimiento físico en el fútbol profesional. Una revisión sistemática
}

The Influence of Ball Possession on Physical Performance

in Professional Football. A Systematic Review

\author{
Carlos Lago-Peñas ${ }^{1}$ (i) \\ Pablo Sanromán-Álvarez’ \\ 1. Universidad de Vigo. Sport Performance Analysis Association, España
}

\begin{abstract}
Resumen
El objetivo de esta revisión sistemática ha sido analizar la influencia de la posesión del balón en el rendimiento físico de los equipos y los jugadores en el fútbol masculino de elite. Se realizó una búsqueda sistemática de artículos relevantes en inglés en una base de datos (Web of Science Core Collection) y un motor de búsqueda (PubMed) siguendo el protocolo de PRISMA (Preferred Reporting Items for Systematic Reviews and Meta-analyses). Las palabras clave "association football" y "soccer" se relacionaron con los términos: "possession of the ball", "possession" "physical activity" y "physical performance". Se buscaron estudios empíricos relacionados con el rendimiento físico y la posesión del balón para su inclusión y análisis. Se revisó el texto completo de 21 estudios. Globalmente, tener un alto (51-66\%) o bajo (34-50\%) porcentaje de posesión del balón produce diferencias muy poco relevantes en las distancias recorridas por los jugadores. Tan sólo la distancia cubierta con y sin la posesión del balón es significativamente diferente. Además, no está claro que correr más o menos pueda asociarse con un incremento de las probabilidades de ganar o perder.
\end{abstract}

Palabras clave: Fútbol, posesión del balón, rendimiento físico, revisión sistemática.

\begin{abstract}
The aim of this systematic review was to provide a summary of empirical research on collective tactical behaviours in football, with a particular focus on organising the methods used and their key fndings. A systematic search of relevant English-language articles was performed on one database (Web of Science Core Collection) and one search engine (PubMed), based on PRISMA (Preferred Reporting Items for Systematic Reviews and Meta-analyses) guidelines. The keywords 'football' and 'soccer' were each paired with all possible combinations of the following keywords: "possession of the ball", "possession" "physical activity" and "physical performance". Empirical studies that were related to physcial performance and possession of the ball were sought for inclusion and analysis. Full-text articles of $2 a$ studies were reviewed. Globally, having either a high (51-66\%) or low (34-50\%) percentage of ball possession results in trivial differences in match-running. There were significant difference in the distance covered by players distance covered by players when the team is in possession compared to out of possession. There is not a clear association between success and the distance covered by players.
\end{abstract}

Keywords: soccer, possession of the ball, physical performance, systematic review.

* Autor de correspondencia: Carlos Lago-Peñas, clagop@uvigo.es

Recibido: 15 de mayo de 2020

Aceptado: 19 de junio de 2020

Publicado: 14 de julio de 2020

Como citar (APA): Lago-Peñas, C., \& Sanromán-Álvarez, P. (2020). La influencia de la posesión del balón en el rendimiento físico en el fútbol profesional. Una revisión sistemática. JUMP, (2), 68-80. https://doi.org/10.17561/jump.n2.7

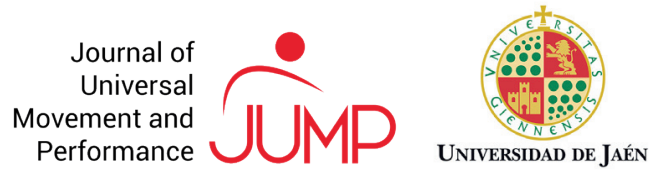




\section{Introducción}

El uso de la tecnología en el fútbol se ha extendido enormemente en los últimos años para medir, controlar y predecir la carga cardiovascular y neuromuscular de las tareas de entrenamiento y la competición (Bradley et al., 2009). El desarrollo de sofisticados sistemas semiautomáticos de cámaras como, por ejemplo, Prozone ${ }^{\circledR}$ y Amisco ${ }^{\circledR}$, hapermitido cuantificardeuna manera muy fiableel rendimiento físico (distancia recorridas, intensidad de carrera, número de acciones de alta intensidad, tiempos de recuperación entre esfuerzos, etc.) y las acciones técnicas individuales y colectivas (pases, entradas, lanzamientos, posesión del balón, etc.) de los futbolistas en situaciones reales de juego. Además, el uso de sistemas de posicionamiento global (GPS), cada vez más habituales no sólo en los equipos de alto nivel sino también en otros de categorías semi-profesionales, permite el seguimiento del comportamiento de los jugadores a tiempo real. A pesar de este extraordinario desarrollo tecnológico que ha incrementado enormemente la capacidad de los entrenadores e investigadores para recoger datos, los factores que explican o modulan las demandas físicas y técnicas del fútbol no se conocen con detalle y requieren de investigaciones.

El desarrollo del juego está influenciado por diversos factores situacionales y ambientales que pueden afectar al comportamiento de los jugadores y los equipos (Lago-Peñas 2012). Dentro de los factores situacionales destacan variables como el sistema de juego de los equipos (Bradley et al., 2009; Carling y Dupont, 2011), la posesión del balón (Lago 2009; Lago y Martin, 2007; Di Salvo et al., 2009), el nivel de los oponentes (Rampinini et al. 2009; Hoppe et al. 2015) y el marcador (Lago Peñas et al., 2010). Dentro de los factores ambientales, se ha demostrado que la altitud (Garvican et al., 2014; Nassis 2013) y la temperatura (Carling et al., 2011) pueden afectar al normal comportamiento de los jugadores al provocar ciertas limitaciones fisiológicas (Waldron y Highton 2014).

Quizás el indicador del rendimiento más popular en el fútbol es la posesión del balón (véase, por ejemplo, a Bate, 1988; Carmichael, Thomas y Ward, 2001; Dawson, Dobson y Gerrard, 2000; Garganta, 2000; Gómez y Álvaro, 2002; Hadley et al., 2000; Hughes y Bartlett, 2002; James, Jones y Mellalieu, 2004; Lago y Dellal, 2010; Lago y Martin, 2007). Sin embargo, a pesar de esta notable producción científica no se conoce con detalle cuál es el impacto de tener la posesión del balón sobre el rendimiento físico de los jugadores. La evidencia científica es limitada y no es concluyente. A pesar de ello, es habitual escuchar a algunos entrenadores decir que tener la posesión del balón reduce la fatiga de los futbolistas. Como se tiene la iniciativa en el juego, se corre menos. Los éxitos de los equipos y selecciones que apuestan por la posesión del balón y el juego combinativo, con jugadores aparentemente poco dotados para desarrollar un juego basado en lo físico, no han hecho más que alimentar esta posición. Sin embargo, cuando se analizan detenidamente las estadísticas post-partido no está tan clara esta idea. Muchos jugadores con una aparente limitada condición física son los que más metros recorren en el partido. Claro que muchas veces lo hacen fuera del espacio que recoge la cámara de televisión cuando filma al balón. ¿Corren menos los equipos que no tienen la pelota? ¿Pueden apostar todos los conjuntos por plantear los partidos desde el dominio del balón? ¿Hay que descartar el juego directo y la defensa sin la pelota en el entrenamiento de los equipos?

En respuesta a estas cuestiones, el objetivo de esta revisión sistemática es proporcionar un resumen de la investigación empírica que ha estudiado la relación entre la posesión del balón y el rendimiento físico en el fútbol de elite. Se trata en definitiva de comprender qué consecuencias físicas y técnico-tácticas tiene para los jugadores y los equipos apostar por la posesión del balón y la iniciativa en el juego.

\section{Método}

\section{Búsqueda de artículos y criterios de inclusión y exclusión}

La revisión sistemática se ha llevado a cabo siguiendo el protocolo de PRISMA (Preferred Reporting Items for Systematic reviews and MetaAnalysis). (Liberati et al., 2009; Moher et al., 2009). Se realizaron las búsquedas en las bases de datos de Web of Science (WOS) y Pubmed relacionando las palabras clave "association football" y "soccer" con los términos: "possession of the ball", "possession" "physical activity" y "physcial performance". La última búsqueda se realizó el 10 de Abril de 2020. 
Los criterios de inclusión de los trabajos incluidos en esta revisión fueron los siguientes: estudios originales, escritos en inglés y que la muestra estuviese conformada por jugadores adultos masculinos de elite. Los siguientes registros fueron eliminados: resúmenes de conferencias y aquellos trabajos que no aportasen detallada sobre el rendimiento físico de los futbolistas.

La evaluación de la inclusión o no de los artículos fue inicialmente realizada por un autor (PS). Todos los documentos identificados fueron seleccionados primero por el título y el abstract (resumen). Los estudios que generaron incertidumbre en la exclusión se retuvieron provisionalmente hasta una revisión posterior del texto completo. Cualquier ambigüedad hacia la inclusión o exclusión de un estudio específico se discutió entre los dos autores de la revisión (PS, CL). Los desacuerdos sobre la inclusión o exclusión final de los estudios se resolvieron por consenso.

\section{Calidad de los estudios}

Una vez seleccionados los estudios para la posterior revisión, la calidad de cada trabajo se evaluó mediante el análisis de una lista de 16 ítems adaptada de otras revisiones previas (Sarmento et al., 2018a y 2018b). Las preguntas estuvieron relacionadas con: (1) claridad del objetivo; (2) relevante revisión de la literatura; (3) diseño de estudio apropiado; (4) muestra del estudio; (5) justificación del tamaño de la muestra; (6) consentimiento informado; (7) fiabilidad de las medidas de resultado; (8) validez de los resultados; (9) descripción detallada del método; (10) informes de resultados; (11) métodos de análisis; (12) descripción de la implicaciones prácticas; (13) descripción de los abandonos (si los hay); (14) conclusiones extraídas adecuadamente; (15) implicaciones para la práctica; y (16) agradecimientos y reconocimiento de las limitaciones del estudio. Estos criterios se puntuaron en una escala binaria $(1=$ sí, $0=$ no), excepto en dos ítems (6 y 13), donde también estaba disponible la opción de "no aplicable". A continuación se calculó la puntuación de cada estudio sumando los puntos totales alcanzados y dividiéndolos por la máxima puntuación posible que el estudio podría haber logrado. La puntuación final se clasificó de la siguiente manera: (1) baja calidad metodológica para puntuaciones $\leq 50 \%$; (2) buena calidad metodológica para puntuaciones entre 51\% y 75\%; y (3) excelente calidad metodológica para puntuaciones $>75 \%$.

\section{Extracción de datos}

De cada uno de los estudios la información más relevante fue recogida por un autor (PS) y revisada por el segundo autor (CL). Los desacuerdos sobre la inclusión o exclusión final de los estudios se resolvieron por consenso. La siguiente información se extrajo de cada uno de los estudios: (1) el autor y el año de publicación del trabajo, (2) la muestra del estudio (partidos, competición, número de jugadores, temporada) (3) el sistema de recogida de datos utilizado, (4) las variables físicas analizadas y (5) los principales resultados.

\section{Resultados}

\section{Resultados de la búsqueda}

La búsqueda inicial recogió 426 artículos. Después de la eliminación de trabajos duplicados, los estudios se redujeron a 200 que fueron posteriormente revisados por su título y resumen para comprobar si cumplían los criterios de inclusión, lo que redujo a 31 los artículos para la revisión. Finalmente, la lectura detallada de estos trabajos eliminó otros 10 estudios, quedando la muestra final en 21 documentos. El diagrama de flujo detallado de este proceso se presenta en la Figura 1.

\section{Calidad de los estudios}

En la evaluación de la calidad metodológica, la puntuación media de los estudios incluidos en la revisión fue del $84 \%$. Uno de los trabajos (Carling et al., 2016) alcanzó la puntuación máxima del 100\%; mientras que ningún estudio obtuvo una puntuación inferior al $75 \%$. Todos los estudios se clasificaron en consecuencia con una excelente calidad metodológica. La fiabilidad interobservadores alcanzó un valor de 0,90, indicando en consecuencia un nivel de acuerdo muy alto entre los observadores. Las principales deficiencias en la calidad metodológica estuvieron relacionadas con los ítems 15 - descripción de la implicaciones prácticas - y 16 - agradecimientos reconocimiento de las limitaciones del estudio - . 


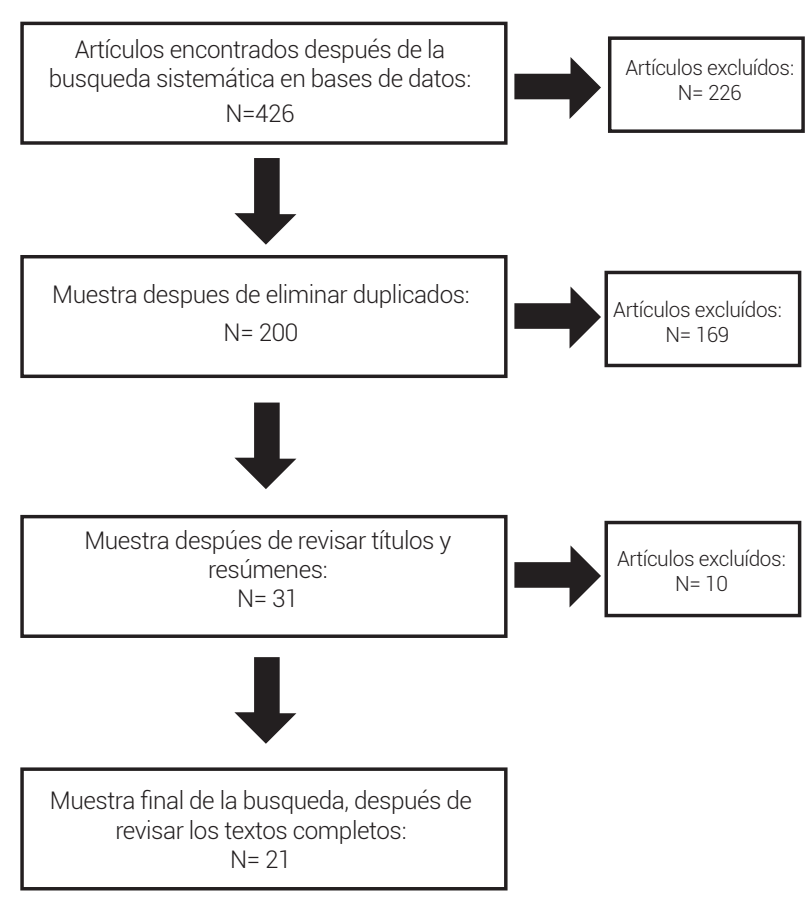

Figura 1. Diagrama de flujo en el proceso de selección de los artículos

\section{Características básicas de los estudios incluidos en la revisión}

Los años de publicación de los artículos van desde 2009 hasta 2019. El origen geográfico de los estudios es el siguiente: Reino Unido $(n=9)$, Francia $(n=4)$, China $(n=2)$, Italia $(n=1)$, Brasil $(n=1)$,
Polonia $(n=1)$, Portugal $(n=1)$, Alemania $(n=1)$, and Italy $(n=1)$. Las competiciones recogidas analizan a La Liga (España), la English Premier League y la Championship English League (Inglaterra), la Ligue 1 (Francia), la Bundesliga (Alemania), la A-League (Australia), la Superliga de China, la Europa League y la Copa Mundial de la FIFA. En todos los casos la muestra incluye a futbolistas profesionales de élite masculinos. Los sistemas recogida de los datos para las investigaciones se han basado fundamentalmente en sistemas semiautomáticos de tracking como AMISCO Pro ${ }^{8}$ (8 estudios), Prozone Sports Ltd®n (7 estudios), Tracab Optical Tracking (2 estudiops), MediaCoach (1 estudio), Vis.Track (1 estudio) y Sics (1 estudio). Un único estudio (Gronow et., 2014) ha utilizado un dispositivo de GPS portátil (PI Pro X, GPSports, Australia, $5 \mathrm{~Hz}$ ).

\section{Resumen de los estudios individuales}

En la Tabla 1 se presenta un resumen individual de todos los estudios revisados. Se proporciona información sobre el autor y el año de publicación del trabajo, la muestra del estudio (partidos, competición, número de jugadores, temporada), el sistema de recogida de datos utilizado, las variables físicas recogidas, los principales resultados y la calidad metodológica del estudio. Los artículos están ordenados alfabéticamente.

Tabla 1. Artículos seleccionados a través del proceso de búsqueda sistemática (orden alfabético)

\begin{tabular}{|c|c|c|c|c|c|}
\hline Autor (año) & Muestra & $\begin{array}{l}\text { Sistema de } \\
\text { recogida de } \\
\text { datos }\end{array}$ & Variables físicas & Resultados principales & $\begin{array}{l}\text { Calidad } \\
\text { metodo- } \\
\text { lógica }\end{array}$ \\
\hline $\begin{array}{l}\text { Ade et al. } \\
\text { (2016) }\end{array}$ & $\begin{array}{l}20 \text { jugadores de un } \\
\text { equipo de la Premier } \\
\text { League inglesa du- } \\
\text { rante las temporadas } \\
2010-2001 \text { a 2013- } \\
2014 \text {. }\end{array}$ & $\begin{array}{l}\text { AMISCO } \\
\text { Pro® }\end{array}$ & $\begin{array}{l}\text { La frecuencia, duración, } \\
\text { distancia cubierta y ve- } \\
\text { locidad media de los } \\
\text { esfuerzos de alta inten- } \\
\text { sidad ( }>21 \mathrm{~km} / \mathrm{h} \text { duran- } \\
\text { te al menos } 1 \text { segundo) } \\
\text { y la recuperación entre } \\
\text { esfuerzos }\end{array}$ & $\begin{array}{l}\text { Los esfuerzos de alta intensidad con balón } \\
\text { y la velocidad promedio de los esfuerzos } \\
\text { fueron mayores en los interiores que en los } \\
\text { centrales, los mediocentros y los delan- } \\
\text { teros (ES }=0.9-2.1, p<0.05) \text {. Los interiores } \\
\text { realizaron esfuerzos más repetidos que los } \\
\text { centrales y los centrocampistas centrales } \\
(E S=0.6-1.3, p<0.05) \text {. }\end{array}$ & $93 \%$ \\
\hline $\begin{array}{l}\text { Andrzejew- } \\
\text { ski et al. } \\
\text { (2014) }\end{array}$ & $\begin{array}{l}10 \text { partidos de la Eu- } \\
\text { ropa League. } 147 \text { ju- } \\
\text { gadores: } 39 \text { defensas } \\
\text { centrales, } 35 \text { latera- } \\
\text { les, } 35 \text { mediocentros, } \\
20 \text { interiores y } 18 \\
\text { delanteros. }\end{array}$ & $\begin{array}{c}\text { AMISCO } \\
\text { Pro® }\end{array}$ & $\begin{array}{l}\text { Distancia total } \\
\text { Distancia alta velocidad } \\
(21,0-24,0 \mathrm{~km} / \mathrm{h}) \\
\text { Distancia mediante } \\
\text { sprints }(>24 \mathrm{~km} / \mathrm{h})\end{array}$ & $\begin{array}{l}\text { Existen diferencias entre los puestos espe- } \\
\text { cíficos en las distintas variables físicas con } \\
\text { y sin el balón. En general, los delanteros son } \\
\text { los más que metr5o recorren a alta inten- } \\
\text { sidad y sprint con y sin balón y los defensas } \\
\text { centrales los que menos distancia recorren. }\end{array}$ & $80 \%$ \\
\hline
\end{tabular}


Tabla 1. Artículos seleccionados a través del proceso de búsqueda sistemática (orden alfabético) (Continuación)

\begin{tabular}{|c|c|c|c|c|c|}
\hline $\begin{array}{l}\text { Bradley et al. } \\
\text { (2009) }\end{array}$ & $\begin{array}{l}28 \text { partidos de la Pre- } \\
\text { mier League ingle- } \\
\text { sa en la temporada } \\
2005-2006 \text {. }\end{array}$ & $\begin{array}{c}\text { Prozone } \\
\text { Sports Ltd® }\end{array}$ & $\begin{array}{l}\text { Distancia total } \\
\text { Distancia mediante } \\
\text { sprint }(>7 \mathrm{~m} / \mathrm{s}) \\
\text { Distancia mediante } \\
\text { alta intensidad }(<5,5- \\
6,9 \mathrm{~m} / \mathrm{s}) \\
\text { Frecuencia de sprints } \\
\text { Frecuencia de esfuer- } \\
\text { zos de alta intensidad }\end{array}$ & $\begin{array}{l}\text { Se constató un descenso en la distancia } \\
\text { recorrida mediante carrera de alta inten- } \\
\text { sidad con }(148 \mathrm{~m} \text { vs } 193 \mathrm{~m}, \mathrm{p}<<0.01) \text { y sin } \\
\text { posesión del balón ( } 229 \mathrm{~m} \text { vs. } 278 \mathrm{~m} \text {, } \mathrm{p} \\
<0.01) \text { entre los últimos } 15 \text { minutos y los } \\
\text { primeros } 15 \text { minutos del partido. }\end{array}$ & $78 \%$ \\
\hline $\begin{array}{l}\text { Bradley et al. } \\
(2013)\end{array}$ & $\begin{array}{l}54 \text { partidos de la Pre- } \\
\text { mier League inglesa. } \\
810 \text { jugadores: } 199 \\
\text { defensas centrales, } \\
177 \text { laterales, } 110 \\
\text { interiores, } 191 \text { medi- } \\
\text { ocentros y } 133 \text { delan- } \\
\text { teros }\end{array}$ & $\begin{array}{l}\text { Prozone } \\
\text { Sports Ltd® }\end{array}$ & $\begin{array}{l}\text { Distancia total } \\
\text { En pie }(0-0.6 \mathrm{~km} / \mathrm{h}) \text {, an- } \\
\text { dando }(0.7-7.1 \mathrm{~km} / \mathrm{h}) \text {, } \\
\quad \text { trotando }(7.2-14.3 \\
\mathrm{km} / \mathrm{h}) \text {, carrera me- } \\
\text { dia }(14.4-19.7 \mathrm{~km} / \mathrm{h}) \text {, } \\
\text { carrera alta intensidad } \\
(19.8-25.1 \quad \mathrm{~km} / \mathrm{h}) \text {, } \\
\text { sprint }(>25.1 \mathrm{~km} / \mathrm{h}) \\
\text { Tiempo de recuperación } \\
\text { entre esfuerzos } \\
\text { Con y sin la posesión } \\
\text { del balón }\end{array}$ & $\begin{array}{l}\text { La distancia total ( } 10690 \pm 996 \text { vs } 10778 \\
\pm 979 \text { m, TE }=0.11) \text { y la distancia de car- } \\
\text { rera de alta intensidad ( } 931 \pm 299 \text { vs } 938 \pm \\
311 \text { m; ES }=0.13 \text { ) fueron similares entre los } \\
\text { EPBA y los EPBB. Sin embargo, la carrera de } \\
\text { alta intensidad con posesión del balón para } \\
\text { los EPBA fue un } 31 \% \text { más alta ( } P<0.01) \text { que } \\
\text { para los EPPB ( } 449 \pm 266 \text { vs } 343 \pm 236 \text { m; } \\
\text { ES = 0.42), pero } 22 \% \text { más baja sin posesión } \\
\text { del balón ( } 423 \pm 153 \text { vs } 539 \pm 177 \text { m; ES = } \\
0,73) \text {. Los defensas centrales de los EPBB } \\
\text { cubrieron un } 33 \% \text { menos (p<0.01) de carre- } \\
\text { ra de alta intensidad con posesión del balón } \\
\text { que los defensas centrales de los EPBA. } \\
\text { Mientras que los mediocentros, delanteros } \\
\text { e interiores de los EPBB cubrieron más dis- } \\
\text { tancia en carreras de alta intensidad sin } \\
\text { el balón y menos con balón que los EPBA } \\
\text { (rango ES de } 0.91-1.23 \text { ). }\end{array}$ & $78 \%$ \\
\hline $\begin{array}{l}\text { Bradley et al. } \\
\text { (2016) }\end{array}$ & $\begin{array}{l}1400 \text { observaciones } \\
\text { de jugadores durante } \\
7 \text { temporadas de la } \\
\text { Premier League in- } \\
\text { glesa (2006-2007 a } \\
2012-2013)\end{array}$ & $\begin{array}{l}\text { Prozone } \\
\text { Sports Ltd® }\end{array}$ & $\begin{array}{l}\text { Distancia total } \\
\text { En pie }(0-0.6 \mathrm{~km} / \mathrm{h}) \text {, an- } \\
\text { dando }(0.7-7.1 \mathrm{~km} / \mathrm{h}) \text {, } \\
\text { trotando }(7.2-14.3 \\
\mathrm{km} / \mathrm{h}) \text {, carrera me- } \\
\text { dia }(14.4-19.7 \mathrm{~km} / \mathrm{h}) \text {, } \\
\text { carrera alta intensidad } \\
(19.8-25.1 \quad \mathrm{~km} / \mathrm{h}) \text {, } \\
\text { sprint }(>25.1 \mathrm{~km} / \mathrm{h}) \\
\text { Con y sin la posesión } \\
\text { del balón }\end{array}$ & $\begin{array}{l}\text { Los equipos clasificados entre el } 5^{\circ} \text { y el } 8^{\circ} \\
\text { puesto demostraron aumentos moderados } \\
\text { en la distancia de carrera de alta inten- } \\
\text { sidad con posesión del balón a lo largo de } \\
\text { las temporadas analizadas ( } p<0.001 ; T E= \\
0.68 \text { ). Para el reto de equipos, el incremento } \\
\text { fue menos pronunciado durante el mismo } \\
\text { período ( }<<0.005 ; E S=0.26,0.41,0.33 \text { ) }\end{array}$ & $83 \%$ \\
\hline $\begin{array}{l}\text { Carling, C. } \\
(2010)\end{array}$ & $\begin{array}{l}28 \text { jugadores de un } \\
\text { equipo de la Ligue } 1 \\
\text { francesa durante } 30 \\
\text { partidos en las tem- } \\
\text { poradas } 2007-008 \text { y } \\
2008-2009 \text {. } \\
\text { En total } 228 \text { observa- } \\
\text { ciones de jugadores } \\
\text { de campo (media } \\
\text { de } 6,5 \text { partidos por } \\
\text { cada futbolista, rango } \\
\text { 1-24) }\end{array}$ & $\begin{array}{c}\text { AMISCO } \\
\text { Pro }{ }^{\circledR}\end{array}$ & $\begin{array}{l}\text { Distancia cubierta en } \\
\text { las siguientes inten- } \\
\text { sidades de carrera: } \\
0.0-11.0 \mathrm{~km} / \mathrm{h}, 11.1- \\
14.0 \mathrm{~km} / \mathrm{h}, 14.1-19.0 \\
\mathrm{~km} / \mathrm{h} \text { y }>19.1 \mathrm{~km} / \mathrm{h} \text { con } \\
\text { y sin la posesión del } \\
\text { balón. }\end{array}$ & $\begin{array}{l}\text { Los jugadores corrieron una distancia total } \\
\text { promedio de } 191+/-38 \mathrm{~m} \text { con la pelota, de } \\
\text { los cuales el } 34.3 \% \text { estaba cubierto a ve- } \\
\text { locidades }>19.1 \mathrm{~km} / \mathrm{h}, 25.6 \% \text { entre } 14.1- \\
19.0 \mathrm{~km} / \mathrm{h}, 12.5 \% \text { entre } 11.1-14.0 \mathrm{~km} / \mathrm{h} \text { y } \\
27.6 \% \text { a }<11.0 \mathrm{~km} / \mathrm{h} \text {. La distancia media } \\
\text { cubierta por posesión fue de } 4.2+/-0.7 \mathrm{~m} \text {, } \\
\text { la velocidad en la recepción de la pelota fue } \\
\text { de } 10.3+/-0.9 \mathrm{~km} / \mathrm{h} \text {, mientras que la veloci- } \\
\text { dad media y máxima durante las carreras } \\
\text { fue de } 12.9+/-1.0 \mathrm{~km} / \mathrm{h} \text { y } 24.9+/-2.4 \mathrm{~km} / \mathrm{h} \text {, } \\
\text { respectivamente. }\end{array}$ & $100 \%$ \\
\hline $\begin{array}{l}\text { Carling et al. } \\
(2011)\end{array}$ & $\begin{array}{l}35 \text { partidos de la Li- } \\
\text { gue } 1 \text { francesa y } 2 \\
\text { partidos de UEFA } \\
\text { Europa League dis- } \\
\text { putados en las tem- } \\
\text { poradas } 2007-2008 \text {, } \\
2008-2009 \text { y 2009- } \\
2010 . \\
11 \text { jugadores, en total, } \\
60 \text { observaciones }\end{array}$ & $\begin{array}{c}\text { AMISCO } \\
\text { Pro }{ }^{\circledR}\end{array}$ & $\begin{array}{l}\text { Distancia total } \\
\text { Distancia cubierta a alta } \\
\text { intensidad ( }>14.4 \mathrm{~km} / \mathrm{h}) \\
\text { Distancia individual to- } \\
\text { tal cubierta con la pos- } \\
\text { esión del balón }\end{array}$ & $\begin{array}{l}\text { La distancia cubierta con posesión individu- } \\
\text { al de la pelota no difirió entre mitades ( } 99 \mathrm{~m} \\
\text { vs. } 96 \mathrm{~m}, \mathrm{p}=0,973) \text {. La distancia cubierta en } \\
\text { la posesión individual de la pelota no varió } \\
\text { en intervalos de } 15 \text { minutos ( } p=0,821) \text {. No } \\
\text { se observaron diferencias entre los partidos } \\
\text { consecutivos en la distancia total ( }(p=0,385) \text {, } \\
\text { la distancia cubierta mediante carrera de } \\
\text { alta velocidad ( } p=0,249) \text { o la cubierta en } \\
\text { posesión individual de la pelote ( }(p=0,347) \text {. }\end{array}$ & $83 \%$ \\
\hline
\end{tabular}


Tabla 1. Artículos seleccionados a través del proceso de búsqueda sistemática (orden alfabético) (Continuación)

\begin{tabular}{|c|c|c|c|c|c|}
\hline $\begin{array}{l}\text { Clemente et } \\
\text { al. (2019) }\end{array}$ & $\begin{array}{l}9641 \text { observaciones } \\
\text { de jugadores de cam- } \\
\text { po (media de 19,7 } \\
\text { partidos por cada fut- } \\
\text { bolista). Temporada } \\
2013-2014\end{array}$ & $\begin{array}{l}\text { Media- } \\
\text { Coach }\end{array}$ & $\begin{array}{l}\text { Distancia total y a dis- } \\
\text { tintas velocidades ( > } \\
14,21 \text { y } 24 \mathrm{~km} / \mathrm{h} \text { ) con } \\
\text { y sin la posesión del } \\
\text { balón }\end{array}$ & $\begin{array}{l}\text { La distancia cubierta con y sin la posesión } \\
\text { del balón mostraron que los equipos clasifi- } \\
\text { cados en la zona media y media-alta cubri- } \\
\text { eron más distancia total } \\
\text { Por encima de } 14 \mathrm{~km} / \mathrm{h} \text {. sin embargo, los } \\
\text { equipos mejor clasificados cubrieron más } \\
\text { distancia a velocidades más altas. Sin em- } \\
\text { bargo, las diferencias no fueron significati- } \\
\text { vas. }\end{array}$ & $83 \%$ \\
\hline $\begin{array}{l}\text { Carling et al. } \\
\text { (2016) }\end{array}$ & $\begin{array}{l}12 \text { jugadores durante } \\
\text { una temporada. En } \\
\text { total } 207 \text { observa- } \\
\text { ciones ( } 17 \text { partidos de } \\
\text { media cada jugador) }\end{array}$ & $\begin{array}{l}\text { AMISCO } \\
\text { Pro® }\end{array}$ & $\begin{array}{l}\text { Distancia cubierta me- } \\
\text { diante carrera de alta } \\
\text { intensidad ( } \geq 19,8 \mathrm{~km} / \mathrm{h} \\
\text { en un intervalo de ti- } \\
\text { empo de } 0,5 \text { segundos) } \\
\text { con y sin posesión del } \\
\text { balón }\end{array}$ & $\begin{array}{l}\text { El coeficiente de variación de la distancia } \\
\text { cubierta mediante carrera de alta inten- } \\
\text { sidad por lo jugadores cuando el equipo } \\
\text { estuvo con/sin el balón y en la posesión } \\
\text { individual de la pelota fue del } 31,5 \%, 26,1 \% \text {, } \\
60,1 \% \text {, respectivamente. }\end{array}$ & $100 \%$ \\
\hline $\begin{array}{l}\text { Da Mota et } \\
\text { al. (2016) }\end{array}$ & $\begin{array}{l}55 \text { partidos de la } \\
\text { Copa del Mundo Ru- } \\
\text { sia } 2018 \text {. } \\
346 \text { jugadores: } 159 \\
\text { defensas, } 65 \text { medi- } \\
\text { ocampistas y } 122 \\
\text { delanteros }\end{array}$ & $\begin{array}{c}\text { Tracab } \\
\text { Optical } \\
\text { Tracking }\end{array}$ & $\begin{array}{l}\text { Distancia total } \\
\text { Distancia a baja }(\leq 11 \\
\mathrm{km} / \mathrm{h}) \text {, media }(11-14 \\
\mathrm{km} / \mathrm{h}) \text { y alta velocidad } \\
(>14 \mathrm{~km} / \mathrm{h}) \text {. } \\
\text { Número de sprints }(>25 \\
\mathrm{km} / \mathrm{h}) \text {. } \\
\text { Velocidad máxima }\end{array}$ & $\begin{array}{l}\text { Aunque los jugadores en EPBA cubrieron } \\
\text { distancias totales y a baja velocidad meno- } \\
\text { res que los EPBB }(P<0,01) \text {, el TE fue trivial. } \\
\text { La distancia cubierta a velocidades medi- } \\
\text { as y altas fue similar. Los jugadores de los } \\
\text { EPBB recorrieron más distancia sin balón, } \\
\text { pero menos con balón }(P<0,01 ; \text { TE grande). }\end{array}$ & $83 \%$ \\
\hline $\begin{array}{l}\text { Dellal et al. } \\
\text { (2011) }\end{array}$ & $\begin{array}{l}600 \text { partidos (1896 } \\
\text { de La Liga y } 4704 \\
\text { de la English Pre- } \\
\text { mier League) } 5938 \\
\text { jugadores: } 2328 \text { de- } \\
\text { fensas centrales, } 344 \\
\text { laterales, } 1972 \text { medi- } \\
\text { ocentros defensivos, } \\
158 \text { mediocentros } \\
\text { ofensivos, } 150 \text { interi- } \\
\text { ores y } 986 \text { delanteros. }\end{array}$ & $\begin{array}{c}\text { AMISCO } \\
\text { Pro® }\end{array}$ & $\begin{array}{l}\text { Distancia total } \\
\text { Distancia alta velocidad } \\
(21,0-24,0 \mathrm{~km} / \mathrm{h}) \\
\text { Distancia mediante } \\
\text { sprints }(>24 \mathrm{~km} / \mathrm{h})\end{array}$ & $\begin{array}{l}\text { El análisis de estos esfuerzos según la } \\
\text { posesión del balón mostró que la actividad } \\
\text { física en ambas ligas era sustancialmente } \\
\text { diferente en las posiciones de juego en el } \\
\text { medio campo. En La Liga, los mediocentros } \\
\text { de ataque cubrieron la mayor distancia en } \\
\text { carreras de alta intensidad cuando su equi- } \\
\text { po estaba en posesión, mientras que este } \\
\text { fue el caso de los interiores en la Premier } \\
\text { League inglesa }\end{array}$ & $78 \%$ \\
\hline $\begin{array}{l}\text { Di Salvo et } \\
\text { al. (2009) }\end{array}$ & $\begin{array}{l}563 \text { jugadores de la } \\
\text { Premier League in- } \\
\text { glesa durante } 3 \text { tem- } \\
\text { poradas. En total, } \\
7355 \text { observaciones } \\
\text { (media de } 8 \text { partidos } \\
\text { por jugador, rango de } \\
1 \text { a } 57 \text { ) }\end{array}$ & $\begin{array}{l}\text { Prozone } \\
\text { Sports Ltd } \AA\end{array}$ & $\begin{array}{l}\text { Distancia cubierta me- } \\
\text { diante carrera de alta } \\
\text { intensidad ( } \geq 19,8 \mathrm{~km} / \mathrm{h} \\
\text { en un intervalo de ti- } \\
\text { empo de } 0,5 \text { segundos) } \\
\text { con y sin posesión del } \\
\text { balón. } \\
\text { Distancia cubierta me- } \\
\text { diante sprint ( } \geq 25,2 \\
\mathrm{~km} / \mathrm{h} \text { en un intervalo de } \\
\text { tiempo de } 0,5 \text { segun- } \\
\text { dos) } \\
\text { Número de sprints }\end{array}$ & $\begin{array}{l}\text { La distancia recorrida mediante carreras de } \\
\text { alta intensidad con la posesión del balón fue } \\
\text { distinta entre todos los puestos específicos, } \\
\text { con los delanteros y los defensas centrales } \\
\text { cubriendo la mayor y menor distancia, re- } \\
\text { spectivamente. Los delanteros y los defen- } \\
\text { sas centrales fueron los que menos distan- } \\
\text { cia cubrieron sin la posesión del balón. }\end{array}$ & $88 \%$ \\
\hline $\begin{array}{l}\text { Gregson et } \\
\text { al. (2010) }\end{array}$ & $\begin{array}{l}485 \text { jugadores duran- } \\
\text { te } 3 \text { temporadas de la } \\
\text { Premier League ingle- } \\
\text { sa. En total } 7281 \text { ob- } \\
\text { servaciones, con una } \\
\text { media de } 10 \text { partidos } \\
\text { (rango de } 2 \text { a } 50 \text { ) por } \\
\text { jugador }\end{array}$ & $\begin{array}{c}\text { Prozone } \\
\text { Sports Ltd® }\end{array}$ & $\begin{array}{l}\text { Distancia cubierta me- } \\
\text { diante carrera de alta } \\
\text { intensidad ( } \geq 19,8 \mathrm{~km} / \mathrm{h} \\
\text { en un intervalo de ti- } \\
\text { empo de } 0,5 \text { segundos) } \\
\text { con y sin posesión del } \\
\text { balón }\end{array}$ & $\begin{array}{l}\text { Los delanteros fueron los que más metros } \\
\text { recorrieron cuando su equipo tuvo la pose- } \\
\text { sión de la pelota, y los que menos su equipo } \\
\text { no tuvo la pelota. Además, los metros cu- } \\
\text { biertos por los jugadores tuvieron una vari- } \\
\text { abilidad más alta (30\%) cuando el equipo } \\
\text { estaba en posesión de la pelota (aproxima- } \\
\text { damente } 30 \% \text { que cuando no tenía la pose- } \\
\text { sión (aproximadamente } 23 \% \text { ) }\end{array}$ & $78 \%$ \\
\hline
\end{tabular}


Tabla 1. Artículos seleccionados a través del proceso de búsqueda sistemática (orden alfabético) (Continuación)

\begin{tabular}{|c|c|c|c|c|c|}
\hline $\begin{array}{l}\text { Gronow et } \\
\text { al. (2014) }\end{array}$ & $\begin{array}{l}36 \text { jugadores de la } \\
\text { Liga Australiana de } \\
\text { Fútbol durante } 14 \\
\text { partidos de la tempo- } \\
\text { rada } 2003\end{array}$ & $\begin{array}{l}\text { SPI Pro X, } \\
\text { GPSports, } \\
\text { Australia, } \\
5 \mathrm{~Hz}\end{array}$ & $\begin{array}{l}\text { Distancia recorrida en } \\
\text { las siguientes veloci- } \\
\text { dades: }<14 \mathrm{~km} / \mathrm{h},>14 \\
\mathrm{~km} / \mathrm{h},>19 \mathrm{~km} / \mathrm{h} \text { y }>24 \\
\mathrm{~km} / \mathrm{h} \text { con y sin posesión } \\
\text { del balón. }\end{array}$ & $\begin{array}{l}\text { La distancia recorrida a }>14 \mathrm{~km} / \mathrm{h} \text { con } \\
\text { posesión fue significativamente menor en } \\
\text { cuartos ganados que en los perdidos, mien- } \\
\text { tras que la distancia cubierta a }>19 \mathrm{~km} / \mathrm{h} \text { y } \\
>14 \mathrm{~km} / \mathrm{h} \text { sin posesión, fue significativa- } \\
\text { mente mayor en cuartos de partido gana- } \\
\text { dos que perdidos. Los delanteros tuvieron } \\
\text { un mayor porcentaje de tiempo dedicado } \\
\text { a actividades }>14 \mathrm{~km} / \mathrm{h} \text {. con posesión del } \\
\text { balón; los defensores tenían un mayor por- } \\
\text { centaje sin posesión y los centrocampistas } \\
\text { tenían el perfil más equilibrado. En general, } \\
\text { en cuartos de partido ganadores, los equi- } \\
\text { pos tenían una mayor cantidad de posesión } \\
\text { y tiempo invertido a }>14 \mathrm{~km} / \mathrm{h} \text { sin posesión } \\
\text { del balón, lo que fue un predictor significa- } \\
\text { tivo de éxito }\end{array}$ & $78 \%$ \\
\hline $\begin{array}{l}\text { Hooppe et } \\
\text { al. (2015) }\end{array}$ & $\begin{array}{l}306 \text { partidos de } \\
\text { la Bundesliga ale- } \\
\text { mana. Temporada } \\
2012 / 2013 \text {. }\end{array}$ & $\begin{array}{l}\text { Vis.Track } \\
\text { System }\end{array}$ & & & $78 \%$ \\
\hline $\begin{array}{l}\text { Morgans et } \\
\text { al. (2014) }\end{array}$ & $\begin{array}{l}6 \text { jugadores pertene- } \\
\text { cientes a un equipo } \\
\text { de la Championship } \\
\text { League inglesa du- } \\
\text { rante una temporada } \\
\text { ( } 37 \text { partidos de madia, } \\
\text { rango de } 27 \text { a 45). }\end{array}$ & $\begin{array}{c}\text { Prozone } \\
\text { Sports Ltd® }\end{array}$ & $\begin{array}{l}\text { Distancia total } \\
\text { Distancia mediante } \\
\text { sprint }(>7 \mathrm{~m} / \mathrm{s}) \\
\text { Distancia mediante } \\
\text { alta intensidad }(<5,5- \\
6,9 \mathrm{~m} / \mathrm{s}) \\
\text { Frecuencia de sprints } \\
\text { Frecuencia de esfuer- } \\
\text { zos de alta intensidad }\end{array}$ & $\begin{array}{l}\text { Ni las distancias de sprint y de alta inten- } \\
\text { sidad ni la frecuencia de los esfuerzos es- } \\
\text { tán relacionadas con la posesión del equipo } \\
\text { (rango de } p=0.27 \text { a } 0.74 \text { ). La distancia total } \\
\text { cubierta se asoció negativamente con la } \\
\text { posesión ( } p=0.008 \text { ). La posesión del balón } \\
\text { se asoció con una distancia total menor du- } \\
\text { rante los partidos. }\end{array}$ & $78 \%$ \\
\hline $\begin{array}{l}\text { Morgans et } \\
\text { al. (2014) }\end{array}$ & $\begin{array}{l}11 \text { jugadores perte- } \\
\text { necientes a un equipo } \\
\text { de la Championship } \\
\text { League inglesa du- } \\
\text { rante una temporada } \\
\text { ( } 47 \text { partidos de madia, } \\
\text { rango de } 32 \text { a 55). }\end{array}$ & $\begin{array}{l}\text { AMISCO } \\
\text { Pro® }\end{array}$ & $\begin{array}{l}\text { Distancia total } \\
\text { Distancia alta velocidad } \\
(19,1-23.0 \mathrm{~km} / \mathrm{h}) \\
\text { Distancia mediante } \\
\text { sprints }(<23 \mathrm{~km} / \mathrm{h})\end{array}$ & $\begin{array}{l}\text { La distancia total cubierta por el equipo } \\
\text { cuando estaba en posesión de balón fue } 72 \\
\mathrm{~m} / \mathrm{min} \text { menor }(p<0,0001 \text { ) que cuando no } \\
\text { tenía la pelota. No hubo diferencias para las } \\
\text { demás variables. }\end{array}$ & $86 \%$ \\
\hline $\begin{array}{l}\text { Rampinini et } \\
\text { al. (2009) }\end{array}$ & $\begin{array}{l}416 \text { partidos de la } \\
\text { Serie A italiana. Se } \\
\text { analizaron a } 186 \text { ju- } \\
\text { gadores. }\end{array}$ & SICS $®$ & $\begin{array}{l}\text { Distancia total } \\
\text { Distancia mediante car- } \\
\text { rera de alta intensidad ( } \\
>14 \mathrm{~km} / \mathrm{h} \text { ) } \\
\text { Distancia mediante car- } \\
\text { rera de muy alta inten- } \\
\text { sidad (> } 19 \mathrm{~km} / \mathrm{h} \text { ) } \\
\text { Distancia total con } \\
\text { balón } \\
\text { Distancia mediante car- } \\
\text { rera de alta intensidad } \\
\text { con balón } \\
\text { Distancia mediante car- } \\
\text { rera de muy alta inten- } \\
\text { sidad con balón }\end{array}$ & $\begin{array}{l}\text { Los equipos con mejores resultados recor- } \\
\text { ren más distancia mediante carrera de alta } \\
\text { intensidad y muy alta intensidad con balón, } \\
\text { mientras que recorren menos distancia en } \\
\text { todas las categorías de rendimiento físico } \\
\text { sin la posesión del balón. }\end{array}$ & $80 \%$ \\
\hline $\begin{array}{l}\text { Schuth et al. } \\
\text { (2016) }\end{array}$ & $\begin{array}{l}6557 \text { observaciones } \\
\text { del rendimiento de } \\
840 \text { jugadores du- } \\
\text { rante las temporadas } \\
2005-2006 \text { a } 2012- \\
2013 \text { de la Premier } \\
\text { League inglesa. }\end{array}$ & $\begin{array}{c}\text { Prozone } \\
\text { Sports Ltd® }\end{array}$ & $\begin{array}{l}\text { Distancia total } \\
\text { En pie }(0-0.6 \mathrm{~km} / \mathrm{h}) \text {, an- } \\
\text { dando }(0.7-7.1 \mathrm{~km} / \mathrm{h}) \text {, } \\
\text { trotando }(7.2-14.3 \\
\mathrm{km} / \mathrm{h}) \text {, carrera me- } \\
\text { dia }(14.4-19.7 \mathrm{~km} / \mathrm{h}) \text {, } \\
\text { carrera alta intensidad } \\
(19.8-25.1 \quad \mathrm{~km} / \mathrm{h}) \text {, } \\
\text { sprint }(>25.1 \mathrm{~km} / \mathrm{h}) \\
\text { Con y sin la posesión } \\
\text { del balón }\end{array}$ & $\begin{array}{l}\text { Los jugadores que intercambiaron desde } \\
\text { la posición de delantero a interior dem- } \\
\text { ostraron una diferencia moderada en la } \\
\text { carrera de alta intensidad sin posesión del } \\
\text { balón (ES }=-0.98)\end{array}$ & $100 \%$ \\
\hline
\end{tabular}


Tabla 1. Artículos seleccionados a través del proceso de búsqueda sistemática (orden alfabético) (Continuación)

\begin{tabular}{|c|c|c|c|c|c|}
\hline $\begin{array}{l}\text { Yi et al. } \\
\text { (2019) }\end{array}$ & $\begin{array}{l}59 \text { partidos de la } \\
\text { Copa del Mundo Ru- } \\
\text { sia } 2018 \text {. }\end{array}$ & $\begin{array}{l}\text { Tracab } \\
\text { Optical } \\
\text { Tracking }\end{array}$ & $\begin{array}{l}\text { Distancia total } \\
\text { Velocidad máxima } \\
\text { Distancia cubierta me- } \\
\text { diante sprint }(>25 \mathrm{~km} / \mathrm{h}) \\
\text { Distancia } 0-7 \mathrm{~km} / \mathrm{h} \\
\text { Distancia } 7-15 \mathrm{Km} / \mathrm{h} \\
\text { Distancia } 15-20 \mathrm{~km} / \mathrm{h} \\
\text { Distancia } 20-25 \mathrm{~km} / \mathrm{h} \\
\text { Distancia }>25 \mathrm{~km} / \mathrm{h}\end{array}$ & $\begin{array}{l}\text { Los equipos caracterizados por un juego } \\
\text { basado en una mayor posesión de balón } \\
\text { cubrieron más distancia en sprints y carre- } \\
\text { ras de alta intensidad (ES }=0.33 \sim 0.47 \text { ) que } \\
\text { los equipos caracterizados por un juego di- } \\
\text { recto. }\end{array}$ & $83 \%$ \\
\hline $\begin{array}{l}\text { Zhou et al. } \\
\text { (2018) }\end{array}$ & $\begin{array}{l}1430 \text { partidos de la } \\
\text { SuperLeague china } \\
\text { durante las tempora- } \\
\text { das } 2012 \text { a } 2017\end{array}$ & $\begin{array}{l}\text { AMISCO } \\
\text { Pro® }\end{array}$ & $\begin{array}{l}\text { Distancia total, con y sin } \\
\text { el balón } \\
\text { Distancia total medi- } \\
\text { ante sprint }(>23 \mathrm{~km} / \mathrm{h}) \text {, } \\
\text { con y sin el balón } \\
\text { Número de sprints } \\
\text { Distancia total medi- } \\
\text { ante carrera de alta } \\
\text { intensidad }(19,1-23 \\
\mathrm{km} / \mathrm{h}) \text {, con y sin el balón } \\
\text { Número de carreras alta } \\
\text { intensidad }\end{array}$ & $\begin{array}{l}\text { Los resultados mostraron que los equipos } \\
\text { ganadores tuvieron valores más altos en la } \\
\text { distancia total con la posesión del balón y } \\
\text { la distancia de carrera de alta intensidad } \\
\text { con la posesión de balón. Los equipos per- } \\
\text { dedores tuvieron promedios significativa- } \\
\text { mente más altos en la distancia total sin } \\
\text { posesión del balón y la distancia mediante } \\
\text { carrera de alta intensidad sin balón. Entre } \\
\text { las variables que discriminan entre equipos } \\
\text { ganadores, empatados y perdedores se in- } \\
\text { cluye a la distancia cubierta con la posesión } \\
\text { del balón }\end{array}$ & $78 \%$ \\
\hline
\end{tabular}

\section{Discusión}

El objetivo de este trabajo ha consistido en revisar la evidencia científica que relaciona la posesión del balón y el rendimiento físico en el fútbol de alto nivel. La discusión de los resultados de los estudios incluidos en esta revisión se organizará en tres apartados distintos: (i) cómo se ha estudiado el efecto de la posesión del balón sobre el rendimiento físico, (ii) cuál es el impacto de tener el balón en la distancia recorrida por los equipos, (iii) cómo afecta la posesión del balón a la distancia recorrida por los futbolistas a distintas intensidades en función de su puesto específico, y (iv) el efecto de la posesión del balón sobre el resultado de los equipos.

\section{Cómo se ha estudiado el efecto de la posesión del balón sobre el rendimiento físico.}

Las distintas investigaciones han estudiado la influencia de la posesión del balón sobre el rendimiento físico de dos formas distintas.

Bradley et al (2013) y Da Mota et al (2016) dividieron respectivamente a los equipos de la Premier League inglesa y a las selecciones que participaron en la Copa el Mundo de 2014 en dos grupos en función del porcentaje de posesión del balón alcanzado en los partidos: equipos con una posesión del balón alta (EPBA) y equipos con una posesión del balón baja (EPBB). Para ello emplearon un análisis de cluster que les permitió identificar un punto de corte en el porcentaje de posesión del balón entre ambos grupos de equipos. Para Bradley et al. (2013) los resultados identificaron al cluster 1 (EPBA) con un $55 \pm 4 \%$ de posesión del balón (rango de 51 a 66\%) y al cluster 2 (EPBB) con $46 \pm 4 \%$ de posesión del balón (rango de 34 a 50\%). Para Da Mota et al. (2016), los resultados identificaron al cluster 1 (EPBA) con un 56,3 $\pm 4,6 \%$ de posesión del balón (rango de 51 a 70\%) y al cluster 2 (EPBB) con 43,9 $\pm 4,5 \%$ de posesión del balón (rango de 30 a 49\%). A partir de esta clasificación analizaron las distancias totales recorridas por los equipos y por los jugadores en función de su puesto específico.

Posiblemente en esta propuesta metodológica existan algunas limitaciones que deban ser resueltas por trabajos posteriores. En primer lugar, la clasificación de los equipos únicamente en dos grupos (EPBA y EPBB) sea excesivamente amplia. Quizás sea necesario dividir a los equipos en más grupos, por ejemplo: equipos con posesión del balón alta, media-alta, media-baja y baja. Los rangos de la posesión del balón en cada categoría propuesta por Bradley et al. (2013) y Da Mota et al. (2016) puede que sean demasiado amplios y no discriminen demasiado bien los estilos de juego de los equipos. En segundo lugar, cabe pensar que la propuesta por la posesión del balón de los equipos puede cambiar a lo largo de los partidos. Así se ha demostrado consistentemente que el marcador es una variable que afecta al comportamiento de los equipos (por ejemplo, Lago, 2007; Lago 
et al. 2010), haciéndolos más o más ofensivos o defensivos dependiendo de si van ganando o perdiendo. Futuros trabajos deberían incluir las variables situaciones (marcador, jugar en casa o fuera y el nivel del oponente) para encontrar los momentos críticos donde se modifica el estilo de juego de los equipos y poder así de una forma más fina el impacto de la posesión del balón sobre el rendimiento físico. En definitiva, puede que la unidad de análisis no sea el partido. Más bien los episodios dentro del juego donde los equipos poseen la iniciativa o están a la expectativa en el juego (Lago-Peñas 2009).

El resto de trabajos que han relacionado la posesión del balón con la distancia recorrida por los jugadores han apostado por un criterio más micro. Los esfuerzos de los futbolistas se calcularon dependiendo de si el equipo estaba o no con la posesión del balón en cada momento. Los sistemas de tracking semiautomáticos existentes en el mercado permiten diferenciar fácilmente entre ambos momentos del juego y proporcionan una medida distinta para el ataque y la defensa de los equipos. Quizás la mayor limitación de esta perspectiva es que adoptar cadajugada como unidad de análisis no permite comprender los cambios estratégicos de los equipos durante los partidos.

Es posible que la decisión de adoptar la perspectiva macro del equipo o micro de la jugada dependa delos objetivos delainvestigación. Cuando se trate de comprender mejor el comportamiento colectivo de un equipo a lo largo de un partido o una serie de encuentros, la propuesta de Bradley et al (2013) y Da Mota et al. (2016) sea la más recomendable. Cuando el objetivo sea el análisis individual de los jugadores o de los puestos específicos, quizás adoptar la perspectiva más micro pueda ser útil. No obstante, en cualquiera de ambas propuestas es recomendable recoger el efecto de las variables situaciones en el partido.

\section{El efecto de la posesión del balón sobre el rendimiento físico de los equipos}

Los estudios que han estudiado el rendimiento físico en función del porcentaje de posesión del balón alcanzado en los partidos no han encontrado diferencias importantes entre ambos grupos de conjuntos en la distancia total o en los metros recorridos distintas intensidades de carrera.

Parece que no existen diferencias en las distancias recorridas a alta intensidad y sprint entre los EPBA y los EPBB (Bradley et al., 2013; Da Mota et al., 2016; Morgans et al., 2014a y 2014b). Sin embargo, se aprecian diferencias significativas en las distancias cubiertas con y sin posesión del balón. Lógicamente, los EPBA recorren más metros con el balón y los EPBB sin la pelota.

Para Bradley et al. (2013), la distancia total (10690 vs 10778) y la distancia de carrera de alta intensidad (931 vs $938 \mathrm{~m}$ ) fueron similares entre los EPBA y los EPBB. Para Da Mota etal. (2016) aunque los jugadores en EPBA cubrieron distancias totales y a baja velocidad menores que los EPBB $(P<0,01)$, el TE fue trivial. La distancia cubierta a velocidades medias y altas fue similar. Sin embargo, ambos estudios encontraron diferencias significativas en la distancia cubierta con y sin posesión del balón. Sin embargo, para Bradley et al. (2013), la carrera de alta intensidad con posesión del balón para los EPBA fue un $31 \%$ más alta que para los EPPB (449 vs 236 m), pero 22\% más baja sin posesión del balón (423 vs 539m). Del mismo modo, para Da Mota el at. (2016) los jugadores de los EPBB recorrieron más distancia sin balón, pero menos con balón.

Estos resultados han sido también verificados por los estudios que han analizado los esfuerzos de los futbolistas se calcularon dependiendo de si el equipo estaba o no con la posesión del balón en cada momento. Así, para Morgans et al. (2014a) la distancia total cubierta por el equipo cuando estaba en posesión de balón fue $72 \mathrm{~m} /$ min menor que cuando no tenía la pelota. No hubo diferencias para las demás variables. En un estudio similar, los mismos autores (Morgans, 2014b) encontraron que ni las distancias de sprint y de alta intensidad ni la frecuencia de los esfuerzos están relacionadas con la posesión del equipo. Únicamente la posesión del balón se asoció con una distancia total menor durante los partidos.

Algunos estudios han analizado el coeficiente de variación de la distancia cubierta mediante carrera de alta intensidad por los jugadores cuando el equipo estuvo con/sin el balón y la posesión individual de la pelota. Los valores fueron del 31,5\%, $26,1 \%$ y $60,1 \%$, respectivamente (Carling et al. 2016). Estos resultados son también confirmados por Gregson et al. (2010). Los metros cubiertos por los jugadores tuvieron una variabilidad más alta (30\%) cuando el equipo estaba en posesión de la pelota (aproximadamente 30\%) que cuando no tenía la posesión (aproximadamente 23\%). 
El efecto de la posesión del balón sobre la distancia recorrida por los futbolistas a distintas intensidades dependiendo del puesto específico

Las diferencias entre puestos específicos son más claras cuando se examina el rendimiento físico respecto a tener o no la posesión del balón (Trewin et al., 2017). Los jugadores atacantes parecen cubrir una mayor distancia de carrera a alta velocidad (hasta un 70\%) cuando el equipo está en posesión del balón en comparación con la situación opuesta (Andrzejewski et al., 2014; Carling 2010; Di Salvo et al., 2009; Gregson et al., 2010; Gronow et al., 2014); mientras que los defensores cubren una mayor distancia de carrera de alta velocidad (hasta un 150\%) cuando su equipo está sin la posesión del balón en comparación con la situación contraria. Además, parece que los delanteros son los jugadores que más metros recorren cuando el equipo está en posesión del balón y los que menos distancia realizan cuando el equipo no tiene la pelota. En esta última situación del juego, son los mediocampistas los más que distancia cubren (Ade et al., 2016; Dellal et al., 2011; Di Salvo et al. 2009; Gregson et al., 2010). Bradley et al. (2013) encontraron que los defensas centrales de los EPBB cubrieron un 33\% menos $(p<0.01)$ de carrera de alta intensidad con posesión del balón que los defensas centrales de los EPBA. Mientras que los mediocentros, delanteros e interiores de los EPBB cubrieron más distancia en carreras de alta intensidad sin el balón y menos con balón que los EPBA (rango ES de 0.91-1.23), Estos resultados pueden explicarse por las funciones de cada puesto específico. Cuando los equipos tienen la posesión del balón necesitan que los delanteros generen espacios libres en el ataque para alcanzar la portería rival; mientras que los defensores deben cubrir esos desplazamientos y recuperar la posesión del balón (Di Salvo et al. 2009). Así, por ejemplo, los jugadores de los equipos con un alto porcentaje de posesión del balón pasan más tiempo en la mitad ofensiva del campo (Da Mota et al. 2016).

\section{El efecto de la posesión del balón sobre el resultado de los equipos}

La asociación entre la distancia recorrida por los equipos con y sin la posesión del balón y los resultados alcanzados en la competición no es concluyente. No está claro que el rendimiento físico tenga un impacto determinante sobre el éxito de los equipos: correr más o menos no puede asociarse con un incremento de las probabilidades de ganar o perder. Así, por ejemplo, en el trabajo de Hoope et al. (2015) se observaron correlaciones significativas entre el rendimiento físico y la posesión del balón (mejor $r=0.77 ; p<0.01$ ). Sin embargo, solo la distancia total cubierta con la posesión de la pelota fue un predictor significativo $(p<0.01)$ y representó el $60 \%$ de la varianza (R2 $=0.60$ ) en los puntos finales alcanzados por los equipos. Para Morgans et al. (2014b) Ni las distancias de sprint y de alta intensidad ni la frecuencia de los esfuerzos están relacionadas con la posesión del equipo (rango de $p=0.27$ a 0.74). Posiblemente, las distintas formas de evaluar el éxito, las distintas competiciones analizadas (competiciones domésticas o europeas, torneos de liga o de eliminación directa) o el tamaño de la muestra puedan explicar esta falta de consenso.

En cualquier caso, los estudios recogidos en esta revisión apuntan a que los equipos con mejores resultados recorren más distancia mediante carrera de alta intensidad y muy alta intensidad con balón, mientras que recorren menos distancia en todas las categorías de rendimiento físico sin la posesión del balón (Clemente et al., 2019; Gronow et al., 2014; Hoope et al., 2015; Rampinini et al., 2009; Zhou et al., 2018). Estos resultados son normales teniendo en cuenta que los equipos que ganan tienen más tiempo la posesión del balón. Así, por ejemplo, Yi et al. (2018) encontró que Los equipos caracterizados por un juego basado en una mayor posesión de balón cubrieron más distancia en sprints y carreras de alta intensidad (ES $=0.33 \sim 0.47$ ) que los equipos caracterizados por un juego directo.

Parece necesaria mucha más investigación que permita encontrar en qué contextos de los partidos la posesión del balón y el rendimiento físico pueden relacionarse claramente con el éxito.

\section{Conclusiones}

Esta revisión presenta las variables físicas, las características de recogida de los datos y los resultados principales de 21 estudios originales que han estudiado la influencia de la posesión del balón en el rendimiento físico de los equipos y los jugadores en el fútbol masculino de elite. 
Globalmente, tener un alto (51-66\%) o bajo (34$50 \%$ ) porcentaje de posesión del balón produce diferencias muy poco relevantes en las distancias recorridas por los jugadores. La única variable que de forma significativa diferencia la actividad de los futbolistas es la distancia cubierta con y sin posesión del balón. La carrera de alta intensidad con posesión del balón para los EPBA fue un 31\% más alta que para los EPPB, pero $22 \%$ más baja sin posesión del balón.

Las diferencias entre puestos específicos son más claras cuando se examina el rendimiento físico respecto a tener o no la posesión del balón. Los atacantes parecen cubrir una mayor distancia de carrera a alta velocidad (hasta un 70\%) cuando el equipo está en posesión del balón en comparación con la situación opuesta; mientras que los defensores cubren una mayor distancia de carrera de alta velocidad (hasta un 150\%) cuando su equipo está sin la posesión del balón en comparación con la situación contraria. Además, los delanteros son los jugadores que más metros recorren cuando el equipo está en posesión del balón y los que menos distancia realizan cuando el equipo no tiene la pelota. Los mediocampistas son los más que distancia cubren sin la posesión del balón.

La asociación entre la distancia recorrida por los equipos con y sin la posesión del balón y los resultados alcanzados en la competición no es concluyente. No está claro que correr más o menos no puede asociarse con un incremento de las probabilidades de ganar o perder.

Esta revisión sistemática tiene algunas limitaciones. En primer lugar, el origen geográfico de la mayoría de los participantes en los estudios se concentra en muy pocos países; los lectores deberían tener cautela en la generalización de los resultados. Además, le presente revisión recoge únicamente investigaciones en inglés y posiblemente estudios publicados en otros idiomas podrían ser relevantes.

\section{Aplicaciones prácticas}

Los resultados de esta revisión sistemàtica muestran como tener la posesión del balón durante más tiempo que el equipo contrario no tiene una clara repercusión en el rendimiento físico de los futbolistas. La distancia total recorrida y la distancia cubierta a alta intensidad y a sprint, la más importante dentro del juego, no es significativamente distinta para los equipos que apuestan por la posesión del balón y los que no. Sin embargo, los requerimientos técnico-tácticos sí parecen ser diferentes. Los jugadores de los equipos que tienen el balón deberían tener un elevado nivel en las habilidades técnico-tácticas del pase y la recepción del balón. Los equipos que tienen altos porcentajes de posesión del balón: (i) dan más pases, (ii) tienen más éxito en ello, (iii) contactan más veces con el balón y reciben más veces la pelota (iv). Los entrenadores deberían tener en cuenta estas evidencias a la hora de seleccionar el planteamiento de juego. Es posible que no todos los equipos puedan jugar a lo mismo. Apostar por la posesión del balón puede ser interesante si se dispone de jugadores para ello. En el caso contrario, será mejor ceder la iniciativa al equipo rival. No obstante, los entrenadores deberían también tener en cuenta que además de depender del plan de juego previsto antes del partido, la posesión del balón tiene mucho que ver con la propia evolución del juego. Los equipos que van perdiendo acaban incrementando la posesión del balón en el partido con el fin de llevar la iniciativa en el juego e intentar recuperar la desventaja existente en el marcador. Cada partido puede tener entonces distintos episodios (iniciativa, expectativa) que el entrenador deberá preparar específicamente cuando crea que el encuentro puede evolucionar hacia esa situación. Además, la posesión del balón y el rendimiento físico no parecen tener una asociación clara con el éxito de los equipos. Todos estos resultados podrían ayudar a los especialistas que trabajan en este ámbito a conocer mejor la carga interna que tiene un partido o una tarea concreta de entrenamiento.

\section{Referencias}

Ade, J., Fitzpatrick, J. \& Bradley, P. S. (2016). High-intensity efforts in elite soccer matches and associated movement patterns, technical skills and tactical actions. Information for positionspecific training drills. Journal of Sports Sciences, 34(24), 2205-2214. https://doi.org/10.1080/02640414.2016.1217343

Andrzejewski, M., Chmura, J. \& Pluta, B. (2014). Analysis of motor and technical activities of professional soccer players of the UEFA Europa League. International Journal of Performance Analysis in Sport, 14(2), 504-523. https://doi.org/10.1080/24748668.2014.11868739

Clemente, J. A., Requena, B., Jukic, I., Nayler, J., Hernández, A. S. \& Carling, C. (2019). Is Physical Performance a Differentiating 
Element between More or Less Successful Football Teams? Sports, 7(10), 216. https://doi.org/10.3390/sports 7100216

Bate, R. (1988). Football chance: Tactics and strategy. In T. Reilly, A. Lees, K. Davids \& W. Murphy (Eds.), Science and football (pp. 293-301). London: E. \& F. N. Spon.

Bradley, P. S., Archer, D. T., Hogg, B., Schuth, G., Bush, M., Carling, C. \& Barnes, C. (2016). Tier-specific evolution of match performance characteristics in the English Premier League: it's getting tougher at the top. Journal of Sports Sciences, 34(10), 980-987. https://doi.org/10.1080/02640414.2015.1082614

Bradley, P. S., Lago-Peñas, C., Rey, E. \& Gomez Diaz, A. (2013). The effect of high and low percentage ball possession on physical and technical profiles in English FA Premier League soccer matches. Journal of Sports Sciences, 37(12), 1261-1270. https://doi.org/10.1080/02640414.2013.786185

Bradley, P. S., Sheldon, W., Wooster, B., Olsen, P., Boanas, P. \& Krustrup, P. (2009). High-intensity running in English FA Premier League soccer matches. Journal of Sports Sciences, 27(2), 159-168. https://doi.org/10.1080/02640410802512775

Carling, C. (2010). Analysis of physical activity profiles when running with the ball in a professional soccer team. Journal of Sports Sciences, 28(3), 319-326. https://doi.org/10.1080/02640410903473851

Carling, C., Bloomfield, J., Nelsen, L. \& Reilly, T. (2008). The role of motion analysis in elite soccer. Sports Medicine, 38(10), 839862. https://doi.org/10.2165/00007256-200838100-00004

Carling, C., Bradley, P., McCall, A. \& Dupont, G. (2016). Match-tomatch variability in high-speed running activity in a professional soccer team. Journal of Sports Sciences, 34(24), 2215-2223. https://doi.org/10.1080/02640414.2016.1176228

Carling, C. \& Dupont, G. (2011). Are declines in physical performance associated with a reduction in skill-related performance during professional soccer match-play? Journal of Sports Sciences, 29(1), 63-71. https://doi.org/10.1080/02640414.2010.521945

Carmichael, F., Thomas, D. \& Ward, R. (2001). Production and efficiency in Association Football. Journal of Sports Economics, 2. 228-243. https://doi.org/10.1177\%2F152700250100200303

Da Mota, G. R., Thiengo, C. R., Gimenes, S. V. \& Bradley, P. S. (2016). The effects of ball possession status on physical and technical indicators during the 2014 FIFA World Cup Finals. Journal of Sports Sciences, 34(6), 493-500 https://doi.org/10.1080/02640414.2015.1114660

Dawson, P., Dobson, S. \& Gerrard, B. (2000). Stochastic frontiers and the temporal structure of managerial efficiency in English soccer. Journal of Sports Sciences Economics, 1, 24-32. https://doi.org/10.1177\%2F152700250000100402

Dellal, A., Chamari, K., Wong, D. P., Ahmaidi, S., Keller, D., Barros, R. \& Carling, C. (2011). Comparison of physical and technical performance in European soccer match-play: FA Premier League and La Liga. European Journal of Sport Science, 17(1), 51-59. https://doi.org/10.1080/17461391.2010.481334

Di Salvo, V., Baron, R., Tschan, H., Montero, F. C., Bachl, N., \& Pigozzi, F. (2007). Performance characteristics according to playing position in elite soccer. International Journal of Sports Medicine 28(03), 222-227. https://doi.org/10.1055/s-2006-924294

Di Salvo, V., Gregson, W., Atkinson, G., Tordoff, P. \& Drust, B. (2009). Analysis of high intensity activity in Premier League soccer. International Journal of Sports Medicine, 30, 205-212. https://doi.org/10.1055/s-0028-1105950

Garganta, J. (2000). Analisis del juego del futbol. El recorrido evolutivo de las concepciones, métodos e instrumentos [Match analysis in soccer. Evolution of concepts, methods and tools.]. Revista de Entrenamiento Deportivo, XIV (2), 6-13.

Garvican, L., Hammond, K., Varley, M., Gore, C., Billaut, F. \& Aughey, R. (2014). Lower running performance and exacerbated fatigue in soccer played at $1600 \mathrm{~m}$. International Journal of Sports Physiology and Performance, 9(3). 397-404. https://doi.org/10.1123/ijspp.2012-0375

Gómez, M. M. \& Álvaro, J. (2002). El tiempo de posesión como variable no determinante del resultado en los partidos de futbol [Ball possession as non-determinant variable for explaining match results.] El Entrenador Español, 97, 39-47.
Gregson, W., Drust, B., Atkinson, G. \& Salvo, V. D. (2010). Matchto-match variability of high-speed activities in premier league soccer. International Journal of Sports Medicine, 37(04), $237-$ 242. https://doi.org/10.1055/s-0030-1247546

Gronow, D., Dawson, B., Heasman, J., Rogalski, B. \& Peeling, P. (2014). Team movement patterns with and without ball possession in Australian Football League players. International Journal of Performance Analysis in Sport, 14(3), 635-651. https://doi.org/10.1080/24748668.2014.11868749

Hadley, L., Poitras, M., Ruggiero, J. \& Knowles, S. (2000). Performance evaluation of National Football League teams. Managerial and Decision Economics, 27(2), 63-70. https://doi.org/10.1002/1099-1468(200003)21:2\%3C63::AID-

Hoppe, M. W., Slomka, M., Baumgart, C., Weber, H. \& Freiwald, J. (2015). Match running performance and success across a season in German Bundesliga soccer teams. International Journal of Sports Medicine, 36(07), 563-566. https://doi.org/10.1055/s-0034-1398578

Hughes, M. D. \& Bartlett, R. (2002). The use of performance indicators in performance analysis. Journal of Sports Sciences, 20, 739-754. https://doi.org/10.1080/026404102320675602

James, N., Jones, P. D. \& Mellalieu, S. D. (2004). Possession as a performance indicator in soccer. International Journal of Performance Analysis in Sport, 4, 98-102. https://doi.org/10.1080/24748668.2004.11868295

Johnston, R. J., Watsford, M. L., Austin, D. J., Pine, M. J. \& Spurrs, R. W. (2015). An examination of the relationship between movement demands and rating of perceived exertion in Australian footballers. The Journal of Strength \& Conditioning Research, 29(7), 20262033. https://doi.org/10.1519/jsc.0000000000000816

Lago-Peñas, C. (2009). The influence of match location, quality of opposition, and match status on possession strategies in professional association football. Journal of Sports Sciences, 27, 1463-1469. https://doi.org/10.1080/02640410903131681

Lago-Peñas, C. (2012). The role of situational variables in analysing physical performance in soccer. Journal of Human kinetics, 35 , 89-95. https://doi.org/10.2478/v10078-012-0082-9

Lago-Peñas, C., Casais, L, Dominguez, E, \& Sampaio, J. (2010). The effects of situational variables on distance covered at various speeds in elite soccer. European Journal of Sport Science, 10(2), 103-109. https://doi.org/10.1080/17461390903273994

Lago-Peñas, C. \& Dellal, A. (2010). Ball possession strategies in elite soccer according to the evolution of the match-score: The influence of situational variables. Journal of Human Kinetics, 25, 93-100. https://doi.org/10.2478/v10078-010-0036-z

Lago-Peñas, C. \& Martin, R. (2007). Determinants of possession of the ball in soccer. Journal of Sports Sciences, 25(9), 969-974. https://doi.org/10.1080/02640410600944626

Liberati, A., Altman, D.G., Tetzlaf, J., Mulrow, C., Gøtzsche, P.C. \& loannidis, J.P.A, (2009). The PRISMA statement for reporting systematic reviews and meta-analyses of studies that evaluate health care interventions: explanation and elaboration. PLoS Medicine, 6(7): e1000100. https://doi.org/10.1371/journal.pmed.1000100

Moher, D., Liberati, A., Tetzlaf, J., Altman, D.G. \& Group P. (2009). Preferred reporting items for systematic reviews and metaanalyses: the PRISMA statement. PLoS Medicine, 6(7): e1000097. https://doi.org/10.1136/bmj.b2535

Morgans, R., Adams, D., Mullen, R., McLellan, C. \& Williams, M. D. (2014a). Technical and physical performance over an English championship league season. International Journal of Sports Science \& Coaching, 9(5), 1033-1042. https://doi.org/10.1260\%2F1747-9541.9.5.1033

Morgans, R., Adams, D., Mullen, R. \& Williams, M. D. (2014b). Changes in physical performance variables in an English Championship League team across the competitive season: the effect of possession. International Journal of Performance Analysis in Sport, 14(2), 493503. https://doi.org/10.1080/24748668.2014.11868738

Nassis, G.P. (2013). Effect of altitude on football performance: analysis of the 2010 FIFA World Cup Data. Journal of Strength Conditioning Research, 27(3):703-707. https://doi.org/10.1519/jsc.0b013e31825d999d 
Rampinini, E., Coutts, A. J., Castagna, C., Sassi, R. \& Impellizzeri, F. M. (2007). Variation in top level soccer match performance. International Journal of Sports Medicine, 28(12), 1018-1024. https://doi.org/10.1055/s-2007-965158

Rampinini, E., Impellizzeri, F. M., Castagna, C., Coutts, A. J. \& Wisløff, U. (2009). Technical performance during soccer matches of the Italian Serie A league: Effect of fatigue and competitive level. Journal of Science and Medicine in Sport, 12(1), $227-$ 233. https://doi.org/10.1016/j.jsams.2007.10.002

Sarmento, H., Clemente, F.M., Araújo, D., Davids, K., McRobert, A. \& Figueiredo A. (2018a). What performance analysts need to know about research trends in association football (20122016): a systematic review. Sports Medicine. 2018; 48(4), 799-836. https://doi.org/10.1007/s40279-017-0836-6

Sarmento, H., Clemente, F.M., Harper, L.D., Costa, I.T., Owen, A. \& Figueiredo A.J. (2018b). Small sided games in soccer - a systematic review. International Journal of Performance Analysis in Sport, 18(5), 1-57. https://doi.org/10.1080/24748668.2018.1517288

Schuth, G., Carr, G., Barnes, C., Carling, C. \& Bradley, P. S. (2016). Positional interchanges influence the physical and technical match performance variables of elite soccer players. Journal of Sports Sciences, 34(6), 501-508.
Shamseer, L., Moher, D., Clarke, M., Ghersi, D., Liberati, A., Petticrew, M. \&. Stewart, L. A. (2015). Preferred reporting items for systematic review and meta-analysis protocols (PRISMA-P) 2015: elaboration and explanation. British Medical JournaL, 349(January), 1-25.

Trewin, J., Meylan, C., Varley, M.C. \& Cronin, J. (2017). The influence of situational and environmental factors on match-running in soccer: a systematic review, Science and Medicine in Football, 1(2), 183-194. https://doi.org/10.1080/24733938.2017.1329589

Waldron, M., Highton, J. (2014). Fatigue and pacing in high-intensity intermittent team sport: an update. Sports Medicine, 44(12) 1645-1658. https://doi.org/10.1007/s40279-014-0230-6

Yi, Q., Gómez, M. A., Wang, L., Huang, G., Zhang, H. \& Liu, H. (2019). Technical and physical match performance of teams in the 2018 FIFA World Cup: Effects of two different playing styles. Journal of Sports Sciences, 37(22), 2569-2577.26-1037. https://doi.org/10.1080/02640414.2019.1648120

Zhou, C., Zhang, S., Lorenzo Calvo, A. \& Cui, Y. (2018). Chinese soccer association super league, 2012-2017: key performance indicators in balance games. International Journal of Performance Analysis in Sport, 18(4), 645-656. https://doi.org/10.1080/24748668.2018.1509254 